\title{
WAFER LEVEL ANTI-STICTION COATINGS WITH SUPERIOR THERMAL STABILITY
}

\author{
W. R. Ashurst, C. Carraro and R. Maboudian \\ Department of Chemical Engineering \\ University of California \\ 201 Gilman Hall, Berkeley, CA 94720, USA \\ tel 510.643.3489, fax 510.642.4778 \\ ashurbr@uclink4.berkeley.edu
}

\begin{abstract}
This paper describes a processing method which allows for the application of a dichlorodimethylsilane (DDMS) anti-stiction monolayer to MEMS on a wafer scale from the vapor phase. This processing method represents significant advantages over the existing liquid based coating technologies (e.g., OTS and FDTS self assembled monolayers (SAMs) [1]) for a variety of reasons. Earlier SAMs, especially FDTS, are susceptible to high levels of particulate contamination. Additionally, SAMs based on OTS or alkene (e.g., octadecene) chemistries suffer from low thermal stability (up to about $225^{\circ} \mathrm{C}$ in air)[2]. However, monolayers formed using DDMS have the unique combination of low particle susceptibility and high thermal stability (upwards of $425^{\circ} \mathrm{C}$ in air) [3]. By utilizing vapor phase (dry) processing, the problems associated with liquid processing such as scale-up, chemical and substrate handling and process control, can be overcome.

We have designed and built a reactor system that allows for the vapor phase deposition of a variety of monolayer systems on both die and wafer levels. Primarily, this system has been used to study the deposition of DDMS monolayers. First, released microstructures are introduced to the vessel. Then in situ cleaning of the microstructures (as well as the reactor vessel) with a down-stream water (or oxygen) plasma is performed. The reactive precursor is dosed and the surface reaction is carried out. The structures are then ready to be removed from the system and tested.

We have evaluated vapor deposited DDMS films in a number of ways. Contact angle analysis, X-ray photoelectron spectroscopy (XPS) and atomic force microscopy (AFM) have been used to characterize the film on $\mathrm{Si}(100)$. Film properties such as work of adhesion and coefficient of static friction were measured from coated micromachine test structures. It is shown that the DDMS monolayer deposited from the vapor phase is quite effective at reducing adhesion and friction.
\end{abstract}

\section{INTRODUCTION}

One major factor that limits the widespread use and reliability of microelectromechanical systems (MEMS) is adhesion [4]. Adhesion is a result of the dominance of surface forces, such as capillary, hydrogen bonding, electrostatic, or van der Waals, over body forces at the micro-scale. When internal restoring forces of microstructures cannot overcome surface adhesive forces, the devices are said to suffer from stiction. Stiction, which can involve both adhesion and friction, can be categorized into two types. The first type, termed release stiction, results from the method used to release the microdevice from the sacrificial layers needed during fabrication. In this case, structures released in liquid etchants, and subsequently oxidized, are dried in such a way that liquid capillary forces, due to the hydrophilic nature of the oxidized surface, are sufficient to draw compliant microstructure surfaces into contact. Once this occurs, the high energy hydrophilic surfaces remain adhered if the microdevice cannot generate enough restoring force to overcome the adhesion.

Travel support has been generously provided by the Transducers Research Foundation and by the DARPA MEMS and DARPA BioFlips programs.

\author{
W. Frey \\ Robert Bosch Corporation \\ Research and Technology Center \\ 4009 Miranda Ave., Palo Alto, CA 94304, USA \\ tel 650.320.2903, fax 650.320.2999 \\ wilhelm.frey@bosch.com
}

The second type of stiction, called in-use stiction, refers to adhesion that occurrs after the release stage, such as when the device is in operation, in storage or improperly handled. As we shall see, it is the in-use stiction that poses the greatest threat to MEMS reliability.

Engineering solutions to the problem of release stiction, reviewed elsewhere $[\mathbf{5 , 6}$, include novel processing techniques such as critical point drying (CPD), freeze sublimation drying and vapor phase etching of sacrificial layers. Other approaches include surface modification techniques such as roughening the structure surfaces to reduce the contact area and changing the water meniscus shape by surface modification.

While the above techniques can be quite effective at the elimination of release stiction, they generally do not prevent in-use stiction. In-use stiction can be quantified by determining the work of adhesion, employing the cantilever beam array (CBA) technique [7] Approaches to reduce in-use stiction fall under two categories, physical and chemical modifications of surfaces $[\mathbf{5 , 6}$. In the physical approach, the surfaces are roughened to reduce the effective contact areas [8]. With this approach, a factor of 20 reduction in apparent surface tension of the roughened polysilicon is reported.

Various types of chemical modifications have been explored as possible anti-stiction treatments, and have been reviewed elsewhere [6]. It is well known that the application of hydrocarbon or fluorocarbon chlorosilane-based SAMs can greatly improve the adhesion properties of micromechanical surfaces. Although these monolayer systems provide many favorable characteristics, they also possess qualities that limit their applicability. Such qualities can be grouped into two types, those that arise due to the chemistry involved, and those that are due to the solution based SAM formation processes. Although chemistry related limitations can be significant, the issues related to the SAM deposition process are what generally limit the widespread application of the SAMs as anti-stiction methods in industry [6]. One limitation is that the coating process is cumbersome because the SAM solution must be freshly made and appropriately conditioned immediately before each coating. This is due to the sensitivity of the SAM solution to ambient humidity, and the ability of the SAM precursors to polymerize. Another difficulty related to the coating process is one of "portability." The large number of process variables, varying expertise and care of operators, as well as the lack of standardized wafer level coating equipment make the success rate of SAM coating processes vary considerably from run to run and laboratory to laboratory. Perhaps the greatest limitation is that of scaling up the process from single dies to whole wafers and eventually multi-wafer cassettes. Much of the work up to this stage has consisted of releasing dies $1 \times 1 \mathrm{~cm}^{2}$. Scaling up the release and coating process to full-wafer level and eventually to multi-wafer cassettes poses substantial engineering challenges.

Recent developments in the chlorosilane-based monolayer technology address some of these issues by performing the coating process in the vapor phase. Vapor phase processing eliminates the use of organic solvents and greatly simplifies handling of the samples. Moreover, the stoichiometry of the precursor molecules can be more precisely controlled. It has been demonstrated that monolayer films that are produced from the precursor tridecafluoro-1,1,2,2- 
tetrahydrooctyltrichlorosilane $\left(\mathrm{CF}_{3}\left(\mathrm{CF}_{2}\right)_{5}\left(\mathrm{CH}_{2}\right)_{2} \mathrm{SiCl}_{3}, \mathrm{FOTS}\right)$ in a low-pressure CVD style reactor exhibit low adhesion energies $[\mathbf{9 , 1 0}]$. Additionally, in-situ plasma cleaning of the sample as well as in-situ measurement of the film growth provide excellent process uniformity, reproducibility and monitoring capability [10].

The dimethyldichlorosilane $\left(\left(\mathrm{CH}_{3}\right)_{2} \mathrm{SiCl}_{2}\right.$, DDMS $)$ monolayer has also been proposed as a promising anti-stiction surface coating for MEMS. Recently, it was found that the DDMS monolayer was quite effective at reducing friction and adhesion, although not quite as effective as OTS $[\mathbf{3 , 1 1}]$. This precursor has several other properties that make it an attractive candidate for vapor phase processing. The DDMS precursor has a lower tendency to polymerize, it has a vapor pressure at room temperature that is much greater than any other chlorosilane precursor used in anti-stiction processes, and for every DDMS molecule that gets hydrolyzed, only two molecules of $\mathrm{HCl}$ will result, as opposed to three from OTS or FDTS. Perhaps the most compelling distinction that DDMS has over OTS is that it has a much greater thermal stability in an oxidizing ambient. In fact, the DDMS films can withstand upwards of $400^{\circ} \mathrm{C}$ whereas OTS films begin to degrade at about $225^{\circ} \mathrm{C}$.

The goal of this work is to develop a dry process which allows for the deposition of an anti-stiction monolayer at the wafer level. The monolayer must survive other post processing steps, such as packaging, and not impede such processes. The coating process must be one that can easily be adapted to industrial settings, have the potential for automatic control, and satisfy uniformity constraints. To accomplish this goals, and in light of the facts above, we turn our attention to the DDMS precursor deposited from vapor phase and build a process around these constraints.

\section{EXPERIMENTAL}

The deposition system consists of a mechanical pump (base pressure $\sim 2 \times 10^{-3}$ torr) connected to a glass-based reaction chamber, which is connected to a reactant delivery subsystem. The chamber is constructed out of glass (or quartz) so that it is easy to modify and clean, and is rather inexpensive. This is a significant departure from earlier efforts. A convectron pressure gauge and thermocouple are used to measure process parameters. Currently, we have designed and constructed two interchangeable custom chambers for this work. The smaller chamber, which measures about 2 inches in diameter, is useful for coating a few dies at the time, while the larger chamber is used for coating a whole 6 in. wafer. The chambers are equipped with perforated electrodes that can be biased to create an in situ DC plasma. The liquid reactants (in this case deionized water, $18 \mathrm{M} \Omega-\mathrm{cm}$ and DDMS, Gelest 99+\%) are loaded into small glass tubes connected by valves to the chamber. A simplified schematic of this system is shown in Fig. 1 and a photograph of the large reactor is shown in Fig. 2. The reactants are degassed before use with three or more freeze-pump-thaw cycles. Results presented in this paper are from samples prepared in both chambers, and we find that the film quality does not depend on the chamber used.

The $\mathrm{Si}(100)$ samples are rinsed in acetone and then cleaned by exposure to ultraviolet light and ozone (UVO) for 15 minutes. Then the samples are treated with concentrated HF for 10 minutes and UVO cleaned again before introduction to the vapor deposition chamber. Micromachine samples are released by etching for 90 minutes in a 1:1 (v/v) mixture of concentrated HF and $\mathrm{HCl}$. The etchant mixture is rinsed away using a funnel aparatus described elsewhere [1] with deionized water until the $\mathrm{pH}$ of the effluent was 7. The water is similarly displaced with isopropanol (IPA). The structures are transported in IPA and dried in a Tousimis Autosamdri 815-B CPD system. The dried micromachine test structures are then introduced to the deposition chamber.

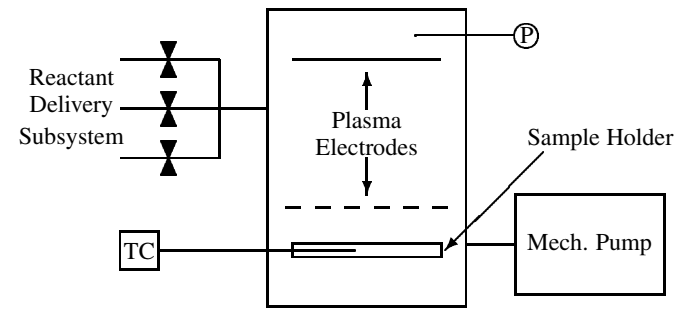

Figure 1. The basic diagram for the vapor-phase anti-stiction film deposition reactor. The sample rests on an aluminum sample holder, and can be heated from below. The plasma electrodes (upper solid, lower perforated) allow for the in situ cleaning of the structures. $(P=$ pressure gauge, $T C=$ thermocouple $)$

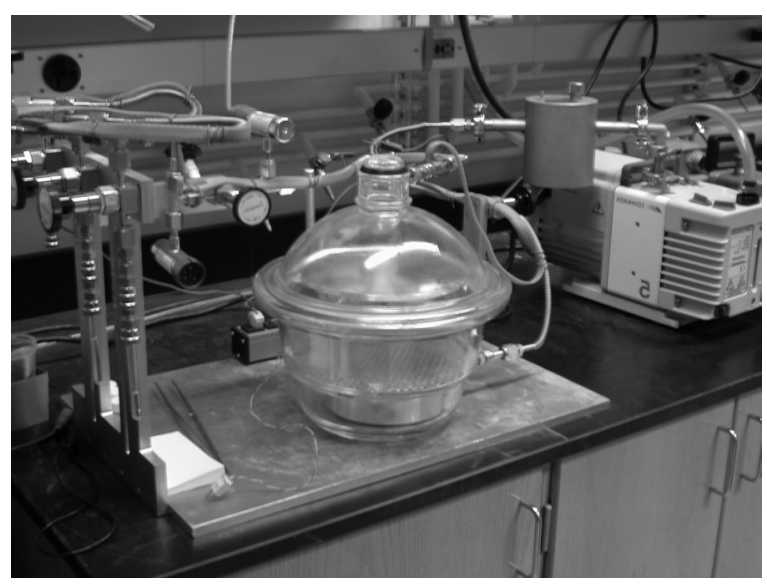

Figure 2. A photograph of the large reactor system.

The samples are placed into the chamber on a large aluminum sample holder that is placed underneath the lower (ground) perforated electrode. The aluminum stage is fitted with a thermocouple near the surface so that temperature can be monitored. Once inside the chamber, micromachine and $\mathrm{Si}(100)$ samples are treated identically, as follows. First, the system is evacuated to a pressure of less than 10 mtorr. Then the samples are cleaned of any organic contamination that usually results from the CPD process by in situ plasma cleaning. This is accomplished by first creating a background of oxygen $\left(\mathrm{O}_{2}\right)$ gas in the chamber by performing purge-pump cycles with $\mathrm{O}_{2}$. Then, $\mathrm{O}_{2}$ is admitted into the chamber so that the pressure of $\mathrm{O}_{2}$ is about 300 mtorr. Next, a downstream plasma is struck by applying a DC bias (about $-700 \mathrm{~V}$ ) to the top electrode while the bottom electrode is held at ground. It is very important that micromechanical structures are not placed inside the plasma discharge - they must be placed downstream of the plasma to avoid detrimental side effects such as curling of structural layers. While the $\mathrm{O}_{2}$ plasma is on, water gas is dosed into the chamber (about 500 mtorr) which, over time, effectively displaces the $\mathrm{O}_{2}$. Exposure of silicon to a water plasma is known to leave the surfaces hydroxyl $(-\mathrm{OH})$ terminated [12]. The entire plasma treatment takes about 15 minutes, after which the samples are ready for surface reaction.

The surface reaction is carried out by first dosing the water. Water gas is admitted to the chamber until the pressure is in excess of 5 torr. Then the chamber is evacuated down to the desired pressure of water, between 1 to 1.3 torr, and isolated from the pump. Next, the DDMS precursor is dosed to the chamber until the total pressure is in the range of 2.5 to 3 torr. The reaction is carried out for 10 to 15 minutes, after which time the chamber is pumped out and vented with nitrogen. The samples are removed and ready for testing.

Static contact angle data are taken with DI water (resistivity 
$18 \mathrm{M} \Omega-\mathrm{cm}$ ) and spectroscopic grade hexadecane (Aldrich Chemical Co.) according to the sessile droplet method with a Ramé Hart 100 A goniometer. A Digital Instruments Nanoscope III atomic force microscope is used in tapping mode to image the surfaces. All micromachine actuation is done under normal laboratory ambient conditions, $20^{\circ} \mathrm{C}$ and $40 \%$ relative humidity.

Adhesion tests are carried out using the cantilever beam array method described elsewhere [7]. Some of the test structures used for adhesion measurements are treated in the large reactor, placed about the chamber so that the spacing would resemble a 6 in. wafer. Coeficient of static friction tests are carried out on sidewall friction testers, coated in the smaller reactor. The details regarding the fabrication and actuation of these devices can be found elsewhere [3]. All liquid phase samples were prepared according to the methods outlined in Ref. [3].

To test the performance of the coating after packaging, some 6 in. wafers containing test devices are processed. The 6 in. silicon cap wafer has structured cavities and bond frames. A low temperature melting seal glass paste is screen printed onto the bond frames, followed by drying and prebaking. Alignment of cap and sensor wafer followed by heating to $430^{\circ} \mathrm{C}$ under $1000 \mathrm{~N}$ bonding force for a couple of minutes leads to hermetic sealing with enclosed application specific pressure adjustable from $<5$ torr through $>1000$ torr.

\section{RESULTS AND DISCUSSION}

In order to test the deposition system and to find the correct process conditions, $\mathrm{Si}(100)$ samples were treated under varying process conditions and examined by contact angle. In the experimental setup described previously, the process variables that could be independently manipulated were substrate temperature, partial pressure of water and DDMS, and time. Early experimentation showed that increasing substrate temperature (over the range of $20^{\circ} \mathrm{C}$ to $50^{\circ} \mathrm{C}$ ), all other parameters being equal, resulted in films that had decreasing water contact angle. It is speculated that this effect is due to a decrease in the adsorption of DDMS and/or water at the reactive surface at higher temperatures. The main result of such temperature experiments is that there is no need to heat the sample.

Other preliminary experiments included process sequences where the reactive precursors were dosed to a given set of partial pressures, evacuated, then dosed again to the same set of partial pressures. It was found that there was virtually no effect of the number of dosing cycles on the contact angle so long as the total time for reaction was the same. In other words, four 2.5 minutes doses are equivalent to a single 10 minute dose. This implies that there is not a substantial amount of depletion of the reactive precursors as the reaction proceeds. Depletion of the reactive precursor was a concern, since the surfaces of the glass reactor (and most of the internal components) are also coated by the DDMS, and thus represent a parasitic loss of reactive precursor.

Through other sets of experimentation, it was found that there were optimum conditions for this process. Various early experiments showed that increasing the pressure of the precursor and water resulted in films with higher contact angles. Also increasing the reaction time resulted in films with higher contact angles. However, the partial pressures and time cannot be increased without bound, as an unfortunate side reaction can occur. The DDMS and water can react to produce a type of viscous liquid, presumably a silicone oil. This liquid can therefore be deposited in a very thin and uniform manner, and cannot be seen on $\mathrm{Si}(100)$ test chips. This thin liquid film has a water contact angle of about $104^{\circ}$ to $106^{\circ}$. If cantilever beams are treated under conditions which generate this liquid, severe adhesion results upon actuation. Indeed, the work of adhesion for beams coated with this liquid is so much greater than that of oxide coated beams that it could not be accurately determined. After peeling up the beams with a probe tip, small patches of a liquid-like substance were found underneath the structures. It therefore became clear that the process window in which a high quality film with no residue could be produced was fairly small, and that a set of optimization experiments were needed. Hence, through a series of such experiments, the parameters described in the experimental section were found to give the best results on $\mathrm{Si}(100)$ and micromachines.

Once suitable deposition conditions were found, coating of microstructures was carried out. Early experimentation with cantilever beam arrays yielded inconsistent results. These inconsistencies were traced to the CPD step. It was found that clean oxide samples (water contact angle $\sim 0^{\circ}$ ) which entered the CPD aparatus had a water contact angle of anywhere between $50^{\circ}$ and $80^{\circ}$ after the drying process, indicating that some contamination had occurred. Also, cantilever beams which entered the CPD aparatus with oxide surfaces emerged with varying adhesion properties which were not oxide-like. With this observation, it was clear that a post drying cleaning step was needed. Keeping in mind the requirements for dry and conformal processing, the plasma cleaning step described in the previous section was chosen and implemented. After treatment of the contaminated samples, the water contact angle was again near $0^{\circ}$, and cantilever beams showed oxide-like adhesion behavior. An additional benefit to the plasma cleaning step is that the reactor walls, and other surfaces, are cleaned of any residue from previous coating runs.

With proper and repeatable deposition methods in place, determination of the film properties began. Water contact angle results on treated $\mathrm{Si}(100)$ can be found in Table 1. The data show that the DDMS films produced either by solution methods or vapor methods are similar in wetability. XPS shows no difference in the chemical make-up of films deposited from solution versus vapor methods. AFM, however, reveals an important difference between the two methods. Figure 3 shows AFM images of solution and vapor deposited DDMS films on $\mathrm{Si}(100)$. It is interesting to note the substantial reduction in particles present on the vapor processed sample with respect to the solution processed sample.

\begin{tabular}{|c|c|c|c|c|}
\hline Coating & $\theta_{\mathrm{H}_{2} \mathrm{O}}$ & $\theta_{\mathrm{HD}}$ & $\begin{array}{c}l_{d} \\
(\mu \mathrm{m})\end{array}$ & $\begin{array}{c}\mathcal{W} \\
\left(\mu \mathrm{J} / \mathrm{m}^{2}\right)\end{array}$ \\
\hline $\begin{array}{c}\text { DDMS } \\
\text { vapor }\end{array}$ & $102^{\circ}$ & $38^{\circ}$ & 510 & 62 \\
\hline $\begin{array}{c}\text { DDMS } \\
\text { liquid }\end{array}$ & $103^{\circ}$ & $38^{\circ}$ & 550 & 45 \\
\hline OTS & $110^{\circ}$ & $38^{\circ}$ & 750 & 12 \\
\hline Oxide & $0-30^{\circ}$ & $0-20^{\circ}$ & $<150$ & 20,000 \\
\hline
\end{tabular}

Table 1. Selected properties of the DDMS monolayer deposited from vapor phase versus liquid phase. OTS and oxide values are given for comparison. Property data are water contact angle, hexadecane contact angle, detachment length, apparent work of adhesion, and coeficient of static friction (sidewall), respectively.

The large size reactor was used to determine the uniformity of the deposition process. A bare 6 in. Si(100) wafer was placed in the reactor and treated (using optimum conditions) with DDMS. The wafer was cleaved through the center and contact angle measurements were performed on a cross wafer strip. Of 18 uniformly spaced measurements, the variation in water contact angle across the 6 in. strip was much less than $1^{\circ}$. This was an encouraging result, since it means that scaling the process to accomodate larger samples is a simple matter of creating a larger reaction chamber.

Some 6 in. processed wafers were also coated. Due to the uni- 


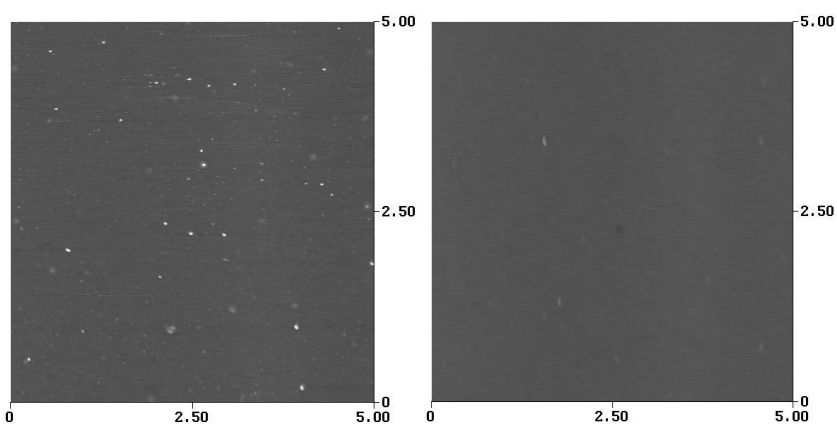

Figure 3. AFM images $(5 \times 5 \mu \mathrm{m})$ of liquid processed (left) and vapor processed (right) DDMS films on Si(100). The particles evident on the liquid processeed sample are not present on the vapor processed sample. The z-range $(10 \mathrm{~nm})$ is the same for both scans.

form coating of the entire wafer, the bonding frames were coated as well. X-ray inspection of bonded wafers show uniform bonding interfaces. A standard process characterization, including leak testing, was performed and showed that the coated wafer exhibited no significant deviation from uncoated wafers. This hermetic bonding through the SAM layer indicates that the monolayer on the bond frame is removed via the lead oxide content of the seal glass. Preliminary stiction behavior characterizations of the packaged test structures qualitatively show substantial reduction in sticking probability. Quantitative statistical characterization is currently underway.

In CBA structures, all beams were found to be free standing after the CPD and DDMS vapor coating process. After actuation, beams of length $450 \mu \mathrm{m}$ or less were consistently free standing, and the detachment length for our test structures, averaged over 20 arrays, was found to be $510 \mu \mathrm{m}$. Using a calculation based on detachment length and other physical parameters [3], the apparent work of adhesion is found to be about $62 \mu \mathrm{J} / \mathrm{m}^{2}$. This value compares well (although slightly higher) with the apparent work of adhesion found for the solution deposited film. Both studies used test structures of the same design from the same wafer, and were actuated in the same manner. In another experiment, several chips containing CBAs were placed at different locations in the large reactor and processed to simulate the full wafer properties. After testing the CBA structures, it was found that the variation of the apparent work of adhesion was no more than that which is present across liquid coated samples.

Sidewall friction testers [3] were used to quantify the coefficient of static friction of the DDMS films. The coated devices were tested, and an average value of 0.35 was found. This value is slightly higher than that for the solution deposited film, but is consistent with the vapor deposited film having lower water conatact angle and higher apparent work of adhesion. Unlike the CBA results, however, the coefficient of static friction showed marked variation from sample to sample, with extremum values at approximately 0.3 to 1.5 times the mean. This was not expected, since all other measurements showed remarkable uniformity. Currently, the reason for this variation is under study.

\section{CONCLUSIONS AND FUTURE WORK}

Based upon the data presented here, a viable vapor phase method for the deposition of the DDMS anti-stiction film has been delveloped. The adhesion and friction properties determined for the vapor phase deposited film are found to be similar to those determined for solution deposited films. However, AFM confirms that fewer particles are generated during the dry coating process than the solution based coating process. Moreover, the scaling issues associated with solution based SAMs are avoided when dealing with dry processes. Also, uniformity tests confirm the process to be quite uniform over an area comparable to a 6 in. wafer. Further plans for the vapor deposition system include investigations on other monolayer systems.

\section{ACKNOWLEDGEMENTS}

The authors wish to thank the NSF (grant DMII-0099765) and Robert Bosch Corporation via the UC-SMART program for project funding. The authors also wish to thank Sandia National Labs for providing test structures. Additional financial support from the National Science Foundation in the form of a graduate fellowship (WRA) is gratefully acknowledged.

\section{REFERENCES}

1. R. Maboudian, W. R. Ashurst, and C. Carraro, "Self-assembled monolayers as anti-stiction coating for mems: Characteristics and recent progress," Sensors and Actuators A, vol. 82, pp. 219-223 (2000).

2. W. R. Ashurst, et al., "Alkene based monolayer films as antistiction coatings for polysilicon MEMS," Technical Digest, Proceedings of the 2000 Solid-State Sensor and Actuator Workshop, Hilton Head, pp. 320-323 (2000).

3. W. R. Ashurst, et al., "Dichlorodimethylsilane as an antistiction monolayer for MEMS: A comparison to the octadecyltrichlorosilane self assembled monolayer," Journal of Microelectromechanical Systems, vol. 10, no. 1, pp. 41-49 (2001).

4. R. Maboudian and R. T. Howe, "Critical review: Adhesion in surface micromechanical structures," J. Vac. Sci. Technol. B, vol. 15, pp. 1-20 (1997).

5. R. Maboudian, "Surface processes in MEMS technology," Surface Science Reports, vol. 30, pp. 207-269 (1998).

6. R. Maboudian, W. R. Ashurst, and C. Carraro, "Tribological challenges in micromechanical systems," Tribology Letters, vol. 12, no. 2, pp. 95-100 (2002).

7. C. H. Mastrangelo, "Adhesion-related failure mechanisms in micromechanical devices," Tribology Letters, vol. 3, no. 3, pp. 223 238 (1997).

8. K. Komvopoulos, "Surface engineering and microtribology for microelectromechanical systems," Wear, vol. 200, pp. 305-327 (1996).

9. J. Sakata, et al., "Anti-stiction silanization coating to silicon microstructures by a vapor deposition process," Transducers '99 (1999).

10. T. M. Mayer, et al., "Chemical vapor deposition of fluoroalkylsilane monolayer films for adhesion control in microelectromechanical systems," Journal of Vacuum Science and Technology B, vol. 18 , pp. 2433-2440 (2000).

11. B. H. Kim, et al., "A new organic modifier for anti-stiction," Journal of Microelectromechaincal Systems, vol. 10, pp. 33-40 (2001).

12. P. R. McCurdy, et al., "A modified molecular beam instrument for the imaging of radicals interacting with surfaces during plasma processing," Review of Scientific Instruments, vol. 68, no. 4, pp. 1684-1693 (1997). 